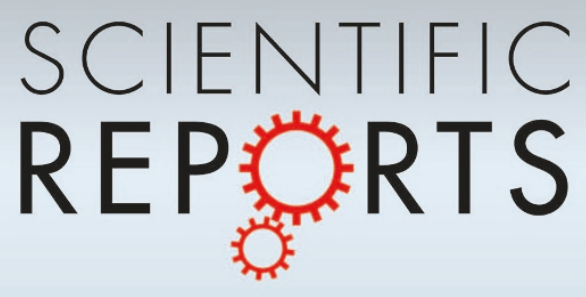

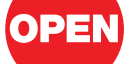

SUBJECT AREAS:

SURFACES, INTERFACES AND THIN FILMS

SPECTROSCOPY

SURFACE SPECTROSCOPY

GEOCHEMISTRY

Received

2 October 2012

Accepted

27 December 2012

Published

15 January 2013

Correspondence and requests for materials should be addressed to

A.N. (nilsson@slac. stanford.edu)

\section{Highly Compressed Two-Dimensional Form of Water at Ambient Conditions}

\author{
Sarp Kaya' , Daniel Schlesinger ${ }^{2}$, Susumu Yamamoto ${ }^{1,5}$, John T. Newberg ${ }^{3,6}$, Hendrik Bluhm ${ }^{3}$, \\ Hirohito Ogasawara' ${ }^{1}$, Tom Kendelewicz ${ }^{4}$, Gordon E. Brown Jr. ${ }^{1,4}$, Lars G. M. Pettersson² \& Anders Nilsson ${ }^{1,2}$
}

'SUNCAT Center for Interface Science and Catalysis, SLAC National Accelerator Laboratory, 2575 Sand Hill Road, Menlo Park, CA 94025, USA, ${ }^{2}$ Department of Physics, AlbaNova University Center, Stockholm University, S-10691 Stockholm, Sweden, ${ }^{3}$ Chemical Sciences Division, Lawrence Berkeley National Laboratory, Berkeley, CA 94720, USA, ${ }^{4}$ Department of Geological \& Environmental Sciences, Stanford University, Stanford, CA 94305-21 15, USA, 5 Synchrotron Radiation Laboratory, The Institute for Solid State Physics, The University of Tokyo, 5-1-5 Kashiwanoha, Kashiwa 277-8581, Japan, 'Department of Chemistry and Biochemistry, University of Delaware, Newark, DE 19716.

The structure of thin-film water on a $\mathrm{BaF}_{2}(111)$ surface under ambient conditions was studied using $\mathrm{x}$-ray absorption spectroscopy from ambient to supercooled temperatures at relative humidity up to $95 \%$. No hexagonal ice-like structure was observed in spite of the expected templating effect of the lattice-matched (111) surface. The oxygen $\mathrm{K}$-edge $\mathrm{x}$-ray absorption spectrum of liquid thin-film water on $\mathrm{BaF}_{2}$ exhibits, at all temperatures, a strong resemblance to that of high-density phases for which the observed spectroscopic features correlate linearly with the density. Surprisingly, the highly compressed, high-density thin-film liquid water is found to be stable from ambient $(300 \mathrm{~K})$ to supercooled $(259 \mathrm{~K})$ temperatures, although a lower-density liquid would be expected at supercooled conditions. Molecular dynamics simulations indicate that the first layer water on $\mathrm{BaF}_{2}(111)$ is indeed in a unique local structure that resembles high-density water, with a strongly collapsed second coordination shell.

W ater is unique in that it exists in several different identifiable amorphous forms, low-density amorphous (LDA), high-density amorphous (HDA), and very-high-density amorphous (VHDA), with a density difference of $33 \%$ between LDA and $\mathrm{VHDA}^{1-4}$. Several metastable amorphous forms have also been observed at various pressures and temperatures ${ }^{5,6}$. It has been proposed that LDA and HDA are glassy forms of low-density liquid (LDL) and high-density liquid (HDL), respectively, in a liquid-liquid transition scenario $^{1,7-10}$, if these liquids would exist as distinct phases. There are many crystalline phases that resemble some of the amorphous forms, but much higher density ices can also be formed under pressure; e.g., ice VII has a density $74 \%$ higher than hexagonal ice $\mathrm{I}_{\mathrm{h}}{ }^{11}$. All of these forms of crystalline ice phases formed at modestly high pressures, as well as amorphous or glassy water, typically have structures in which there is an inward collapse of the $2^{\text {nd }}$ coordination shell. Similarly, water under pressure at room temperature responds through a continuous collapse of the $2^{\text {nd }}$ shell with increasing pressure ${ }^{12}$ where the extrapolated pair-correlation function (PCF) of HDL water has a broad $2^{\text {nd }}$-shell distribution in between those of $\mathrm{HDA}$ and $\mathrm{VHDA}^{2}$; a similar structural rearrangement has been observed in simulations of water in silica nano-confinement ${ }^{13}$. Water thus seems to have the ability to exhibit rather dramatic structural differences with a large range of densities and only minor differences in enthalpy ${ }^{1}$. Here we demonstrate that it is possible to generate an extremely compressed HDL-like form of water at ambient conditions at the $\mathrm{BaF}_{2}(111) /$ water interface, which acts as the pressurizing medium.

The influence of the substrate on interfacial water has often been discussed in terms of ice-like and liquid-like structural signatures ${ }^{14-17}$. In this respect, $\mathrm{BaF}_{2}(111)$ terminated by $\mathrm{F}^{-}-\mathrm{Ba}^{2+}-\mathrm{F}^{-}$trilayers, with a surface lattice parameter of $4.39 \pm 0.1 \AA$, is very close to that of the basal plane of ice $I_{h}(4.52 \AA)$. Thus, an epitaxial layer of ice growth is expected to form on $\mathrm{BaF}_{2}(111)$ at ambient conditions. Although earlier optical absorption experiments suggested the formation of ice-like overlayers under low temperature ${ }^{18}$ and ambient conditions ${ }^{19}$, theoretical estimates of the stability of the epitaxial ice layers are not consistent with these experimental observations ${ }^{20}$. Also in nano-confinement Raman scattering experiments on water have found a non-freezing water layer at the water/ solid interface down to extremely low temperature ${ }^{21}$. Arguments about the stability of epitaxial wetting layers based on molecular dynamics simulations ${ }^{22}$ have not been corroborated because of the effects of surface defects $^{23-25}$. Theoretical calculation $\mathrm{s}^{20}$ using the ASP-W2K potential find an adsorption energy of $-39.78 \mathrm{~kJ} /$ mol for an isolated water molecule on $\mathrm{BaF}_{2}$. Higher coverages resulted in a bilayer hexagonal ice film with an 
increase of the adsorption energy to $-51.4 \mathrm{~kJ} / \mathrm{mol}$, which, albeit higher than the enthalpy of condensation of bulk water $(-44 \mathrm{~kJ} /$ mol), demonstrates only a weak trend towards wetting.

In the present study, the molecular structure of thin-film water under ambient conditions (up to 2.4 monolayers (ML)) as well as ice under low-temperature conditions in ultra-high vacuum (UHV) were elucidated on $\mathrm{BaF}_{2}(111)$ using $\mathrm{x}$-ray absorption spectroscopy (XAS) and $\mathrm{x}$-ray photoelectron spectroscopy (XPS). O K-edge XAS is a powerful local probe for identifying the structures of water in solid and liquid phases; the spectral features are directly related to the hydrogen-bonding environments ${ }^{26}$. The experimental data are complemented with molecular dynamics (MD) simulations, where the first interfacial layer indicates a very high local density $\left(1.23 \mathrm{~g} / \mathrm{cm}^{3}\right)$ and the $\mathrm{O}-\mathrm{O}$ pair-correlation function shows a near-complete collapse of the $2^{\text {nd }}$ shell, well beyond what is seen in HDL and close to merging with the first shell to form a two-dimensional hexagonal structure.

\section{Results}

In order to characterize the structure of the thin water films we used $\mathrm{O} \mathrm{K}$-edge XAS to obtain spectral signatures indicative of specific hydrogen-bonding configurations. The obtained spectra of thin layers of liquid water, however, do not exhibit the expected templating effect resulting in hexagonal ice, but instead show strong similarity to high-pressure ices, such as ice VII with a density of $1.6 \mathrm{~g} /$ $\mathrm{cm}^{3}$. XAS spectra measured at 1.5 Torr water at $30 \%(288 \mathrm{~K}), 60 \%$ $(270 \mathrm{~K})$, and $90 \%(259 \mathrm{~K})$ relative humidity $(\mathrm{RH})$, with the polarization vector of the incident $\mathrm{x}$-ray out-of-plane and in-plane, are shown in Figs. 1(a and 1(b)), respectively. These films correspond to $1.2,1.8$, and $2.4 \mathrm{ML}$ coverage of water (see Supplementary Information for details). At all RHs studied, the XAS spectral features are rather similar: strong pre-edge and weak post-edge features are observed, indicating that, irrespective of the polarization vector orientation and the temperature ( 300-259 K), the structure of thinfilm water on $\mathrm{BaF}_{2}(111)$ does not depend on coverage. At $30 \% \mathrm{RH}$, a monolayer of water forms, as seen in the uptake measurements.

The pre-edge feature at $535 \mathrm{eV}$ has been proposed to be mainly related to weakened $\mathrm{H}$-bond $\mathrm{s}^{26,27}$ whereas the post-edge feature appearing at $540-542 \mathrm{eV}$ is due to $\mathrm{H}$-bonds and is particularly strong for tetrahedrally $\mathrm{H}$-bonded water as in hexagonal ice (ref. 26,28 and

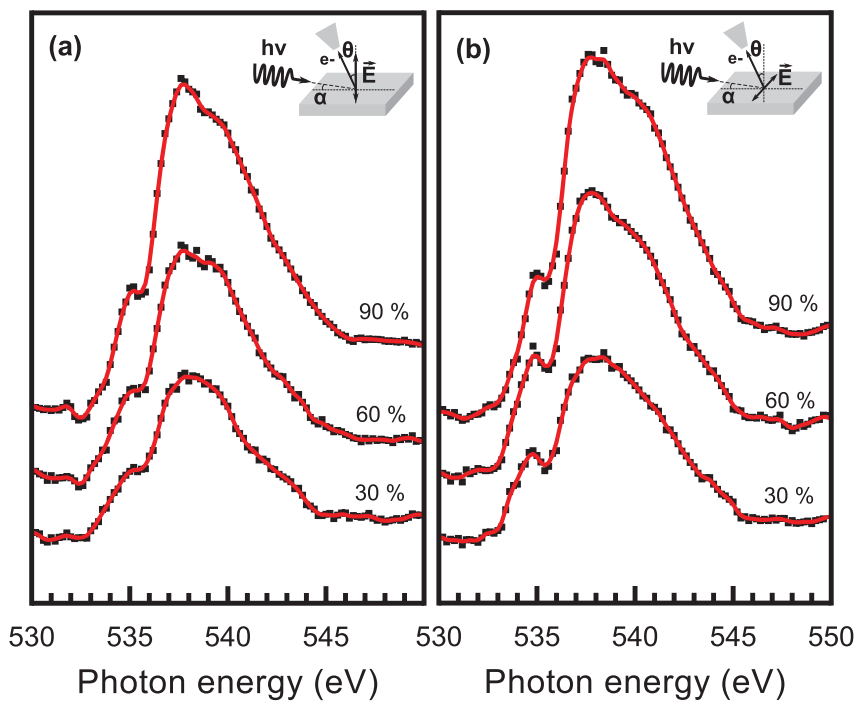

Figure $1 \mid$ XAS spectra of water on $\mathrm{BaF}_{2}(111)$ at 1.5 Torr water pressure with the polarization vector (a) perpendicular and (b) parallel to the surface plane. RH was varied from $30 \%$ to $90 \%$. The incident angle $(\alpha)$ of in-plane polarized light and the take-off angle $(\theta)$ are $\sim 10^{\circ}$ and $35^{\circ}$, respectively. discussions therein regarding various interpretations). The mainedge feature, at $\sim 537 \mathrm{eV}$ is enhanced upon formation of $\mathrm{HDA}^{29}$ and various crystalline high-pressure ice phases such as $\mathrm{I}_{\mathrm{II}}, \mathrm{I}_{\mathrm{VI}}, \mathrm{I}_{\mathrm{VII}}$, and $\mathrm{I}_{\mathrm{VIII}}{ }^{30}$. This enhancement is related to the fact that the second shell collapses from the tetrahedral distance of $4.52 \AA$ in ice $I_{h}$ to much shorter distances, leading to an overall higher density; note that this also slightly increases the pre-edge intensity.

In Fig. 2a, we compare the XAS spectrum of thin-film water on $\mathrm{BaF}_{2}(111)$ at $90 \% \mathrm{RH}$ with the spectrum of crystalline ice formed at low pressures $\left(\mathrm{I}_{\mathrm{h}}\right.$ or $\left.\mathrm{I}_{\mathrm{c}}\right)$ on $\mathrm{BaF}_{2}(111)$ and with the relative change between ice $\mathrm{I}_{\mathrm{h}}$ and $\mathrm{I}_{\mathrm{VII}}{ }^{30}$. The latter corresponds to a high-pressure crystalline ice phase with a density of $1.60 \mathrm{~g} / \mathrm{cm}^{3}$. First, we note that the thin water film XAS spectrum lacks the post-edge feature which is seen in crystalline ice. Furthermore no low pressure ice-like spectral features were observed at any coverage as $\mathrm{RH}$ was increased from $30 \%$ to $90 \%$, even at temperatures as low as $259 \mathrm{~K}$. Considering the arguments of surface-templated ice formation, the templating effect of $\mathrm{BaF}_{2}(111)$ on the structure of the water film is expected to be strongest under this condition. Secondly, we instead note that the water thin-film spectrum has a close resemblance with the highpressure spectrum of ice $\mathrm{I}_{\mathrm{VII}}$.

In Fig. 2b, we note that the XAS spectrum of thin-film water on $\mathrm{BaF}_{2}(111)$ at ambient conditions more closely resembles the spectrum of liquid water than that of low-density crystalline ice on $\mathrm{BaF}_{2}$, as seen from the weak post-edge and strong main-edge and pre-edge features. Because the post-edge is enhanced for tetrahedral $\mathrm{H}$-bonding configurations, we infer that low-density crystalline ice-like local arrangements are very sparse in the water layer on $\mathrm{BaF}_{2}(111)$. This inference is quite surprising because the substrate temperature of $259 \mathrm{~K}$ is deep in the supercooled state of water, and the driving force

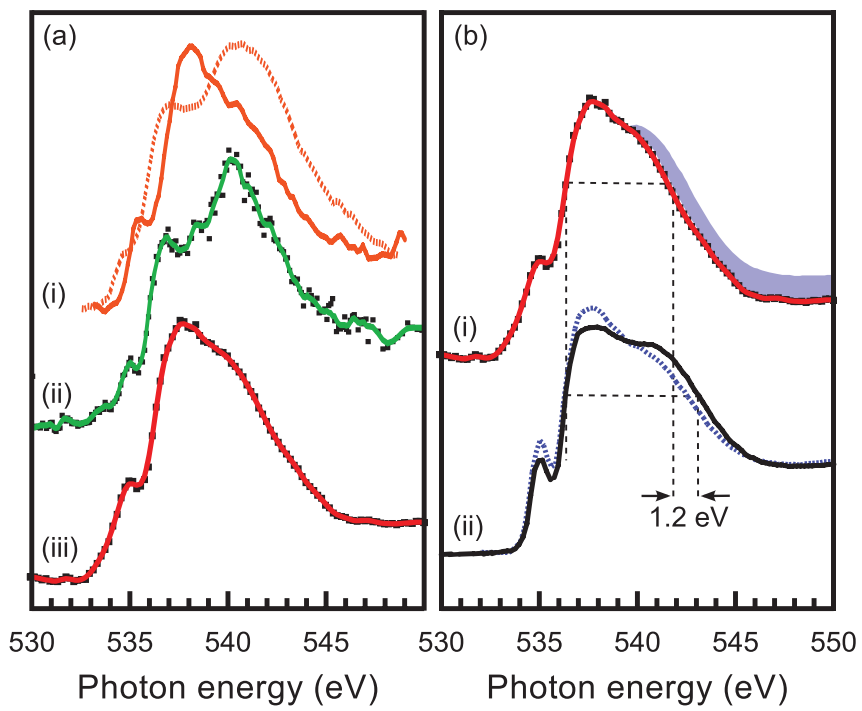

Figure $2 \mid$ (a) Comparison of XAS spectra of (i) bulk ice $\mathrm{I}_{\mathrm{h}}$ (dashed), ice VII $\left(\mathrm{I}_{\mathrm{VII}}\right)$ (solid) ${ }^{30}$ with the spectra of (ii) $2 \mathrm{ML}$ crystalline ice and (iii) supercooled water on $\mathrm{BaF}_{2}(111)$ at 1.5 Torr at $259 \mathrm{~K}(90 \% \mathrm{RH})$. Crystalline ice on $\mathrm{BaF}_{2}(111)$ was prepared by annealing $10 \mathrm{ML}$ LDA to $175 \mathrm{~K}$. XAS spectra of crystalline ice on $\mathrm{BaF}_{2}(111)$ were recorded via exciting with in-plane polarized light. (b) Comparison of XAS spectra of (i) supercooled water on $\mathrm{BaF}_{2}(111)$ at 1.5 Torr at $259 \mathrm{~K}(90 \% \mathrm{RH})$ with (ii, solid) liquid water, and (ii, dashed) $6 \mathrm{M} \mathrm{NaCl}$ solution measured at room temperature ${ }^{31}$. The supercooled water spectrum is an average of two spectra obtained using in-plane and out-of-plane polarizations, shown in Fig. 1. The shaded area represents the difference between bulk ambient liquid and thin-film water. XAS spectra of liquid water and the $6 \mathrm{M} \mathrm{NaCl}$ solution were recorded in transmission mode ${ }^{31}$. The sample was embedded between two $\mathrm{Si}_{3} \mathrm{~N}_{4}$ windows separated by a $300 \mathrm{~nm}$ thick spacer. All spectra are normalized by area. 
for tetrahedral structuring should be rather strong according to expectations from thermodynamics arguments that enthalpy and entropy of water films converge to values typical of ice ${ }^{19}$. Contrary to these enthalpy and entropy estimates from quantification of IR spectral features, the tetrahedral structure of ice for water films on $\mathrm{BaF}_{2}(111)$ has not been found to be the most favorable based on theoretical calculations ${ }^{20}$ and polarizability measurements ${ }^{25}$.

Even more surprising is the observation that, due to the loss of intensity in the post-edge region, the spectral width spanned by the main-edge and post-edge peaks of supercooled water on $\mathrm{BaF}_{2}(111)$ is somewhat smaller than that for liquid water. In Fig. $2 b$ we also compare the thin-film water spectrum with the spectrum of $6 \mathrm{M}$ $\mathrm{NaCl}$ solution at room temperature ${ }^{31}$ and note in the small post-edge a stronger similarity for thin-film liquid on $\mathrm{BaF}_{2}(111)$ with the $\mathrm{NaCl}$

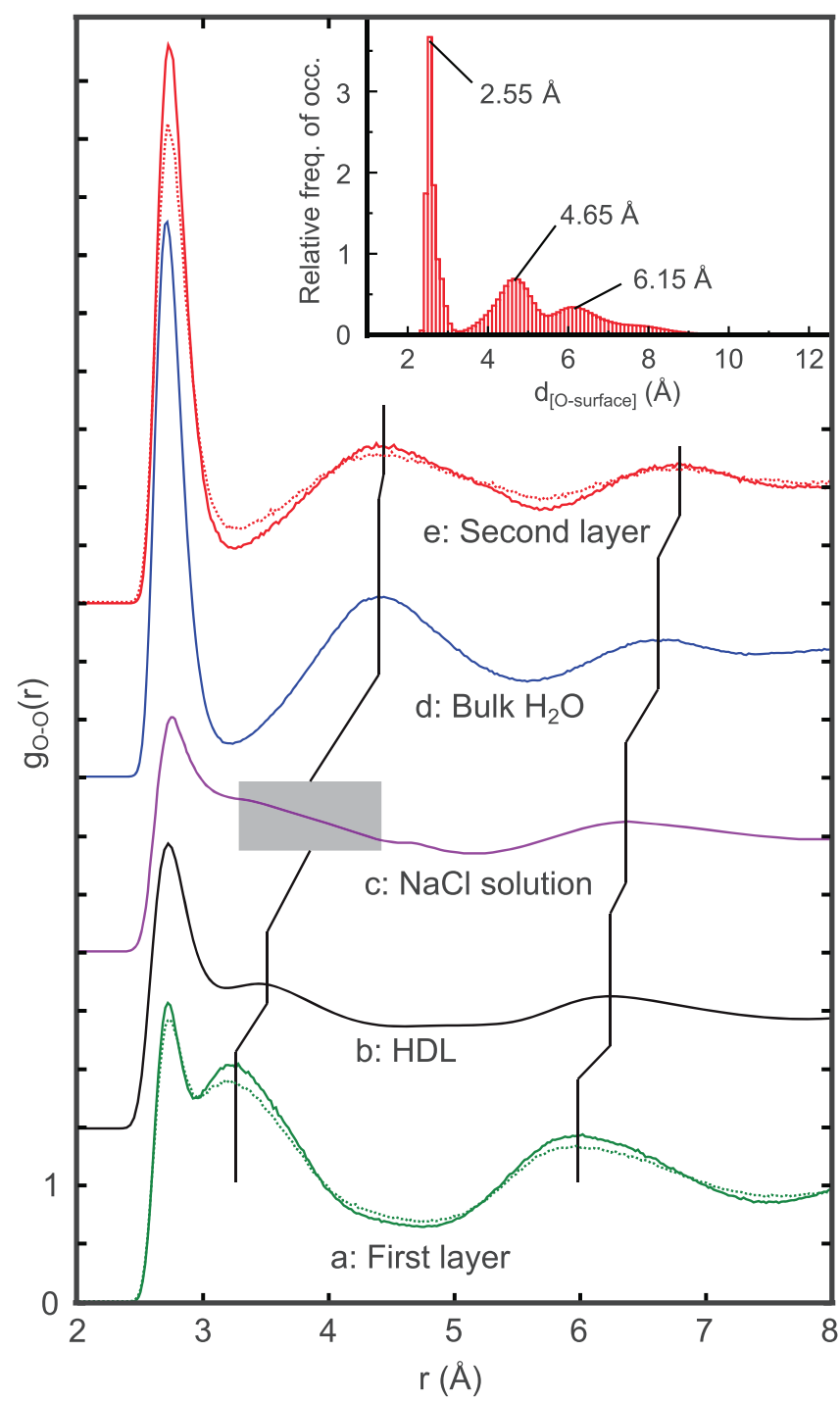

Figure 3 Oxygen-oxygen pair-correlation functions (a) calculated within the first $1 \AA$ layer of $\mathrm{H}_{2} \mathrm{O}$ on the $\mathrm{BaF}_{2}(111)$ surface from MD simulations, full line $\mathrm{T}=260 \mathrm{~K}$, dotted line $\mathrm{T}=290 \mathrm{~K}$; (b) of $\mathrm{HDL}^{34}$; (c) of a $\mathrm{NaCl}$ solution of concentration $1: 10$ salt to water mole ratio ${ }^{44}$; (d) calculated within a $1 \AA$ layer of bulk $\mathrm{H}_{2} \mathrm{O}$ from MD simulation at $\mathrm{T}=260 \mathrm{~K}$; (e) calculated within the second $1 \AA$ layer of $\mathrm{H}_{2} \mathrm{O}$ on the $\mathrm{BaF}_{2}(111)$ surface from $\mathrm{MD}$ simulations, full line $\mathrm{T}=260 \mathrm{~K}$, dotted line $\mathrm{T}=290 \mathrm{~K}$. Note that the pair-correlation functions calculated within a $1 \AA$ layer of water (a, d, e) are normalized differently than in (b, c), cf. Supplementary Information. Inset: Histogram of distances between the oxygen atoms of $\mathrm{H}_{2} \mathrm{O}$ and the surface atoms. solution than with bulk water. In alkaline aqueous solutions the interaction of water with the alkali cations is weak ${ }^{26,31}$, while neutron diffraction measurements ${ }^{32}$ indicate that the resulting structure is still similar to that of water under pressure, which is also supported by $\mathrm{MD}$ simulations ${ }^{33}$. The close spectral similarity of the thin-film water spectrum and the spectra of the $\mathrm{NaCl}$ solution and $\mathrm{I}_{\mathrm{VII}}$ indicates that the water structure should resemble more a HDL-related structure as typically seen upon applying pressure on water with an inward collapse of the $2^{\text {nd }}$ shell.

From the MD simulations, we find a strong layering of water molecules parallel to the surface. The inset of Fig. 3 shows a dramatic localization of the first water layer; the distribution of the molecules within subsequent layers is broader, but the influence of the $1^{\text {st }}$ layer on the $2^{\text {nd }}$ and $3^{\text {rd }}$ layers is evident. To further determine the structure within these layers, we calculated oxygen-oxygen PCFs within layers of defined thickness perpendicular to the surface and compared the calculated PCFs with the experimental PCFs of HDL water ${ }^{34}, \mathrm{NaCl}$ solution $^{31}$ and the PCF of a slab of bulk TIP4P/2005 water of the same thickness as for water on $\mathrm{BaF}_{2}$ (Fig. 3). The first three peaks in the TIP4P/2005 bulk water PCF represent the $1^{\text {st }}, 2^{\text {nd }}$, and $3^{\text {rd }}$ coordination shells located at $2.75,4.4$, and $6.7 \AA$, respectively, in this model. The $1^{\text {st }}$ layer of $1 \AA$ thickness of thin-film TIP4P/2005 water on $\mathrm{BaF}_{2}(111)$, on the other hand, shows a significantly altered structure compared to the $2^{\text {nd }}$ and $3^{\text {rd }}$ layers of the same thickness. The $2^{\text {nd }}$ coordination shell of the $1^{\text {st }}$ layer is collapsed as in the case of $\mathrm{HDL}^{34}$, and the $3^{\text {rd }}$ peak shifted inwards to $6 \AA$, indicating that the $3^{\text {rd }}$ coordination shell adapts to the high-density profile. The $2^{\text {nd }}$ and $3^{\text {rd }}$ layers of the thin film, on the other hand, show a structure similar to that of the bulk. For $\mathrm{T}=260 \mathrm{~K}$, the structure is slightly more pronounced than for $\mathrm{T}=290 \mathrm{~K}$, but does not change qualitatively. These findings are universal and do not change with the different

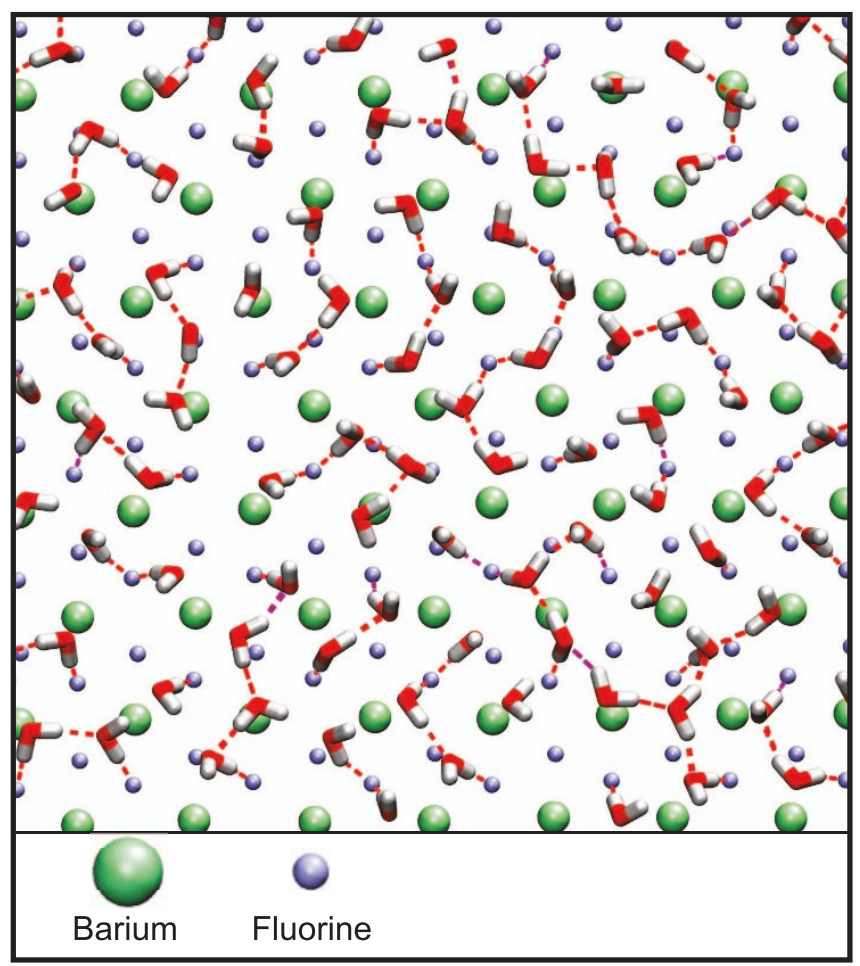

Figure $4 \mid$ A snapshot of the first water layer on $\mathrm{BaF}_{2}(111) . \mathrm{F}^{-}$and $\mathrm{Ba}^{2+}$ ions are color-coded by purple and green, respectively. Red and white sticks represent oxygen and hydrogen atoms of water molecules. Intermolecular hydrogen bonding and water- $\mathrm{BaF}_{2}(111)$ bonding are indicated by dashed lines. $\mathrm{H}$-bond definition: $\mathrm{O}-\mathrm{O}$ distance $<3 \AA$, O-O-H angle $<30$ deg. 
force fields commonly utilized to simulate the properties of water (see Supplementary Information for details).

In Fig. 4 we show a snapshot from the simulation with a strong predominance of chain-like conformations in the layer in contact with the substrate. In comparison to low density crystalline ice, the structure of the $1^{\text {st }}$ layer is more similar to close packing at the expense of breaking $\mathrm{H}$-bonds. The density in the $1^{\text {st }}$ layer is estimated to be $1.23 \mathrm{~g} / \mathrm{cm}^{3}$ (see Supplementary Information for details), which is similar to the density of VHDA; this similarity also fits well with the $2^{\text {nd }}$-shell distance of $3.3 \AA^{1,4}$. However, there are more severe distortions from tetrahedral coordination in the $1^{\text {st }}$ shell in comparison to VHDA which we attribute to the thermal fluctuations prominent near room temperature.

\section{Discussion}

Previous surface $\mathrm{x}$-ray scattering and diffraction studies of the watersilica ${ }^{35}$ and water- $\mathrm{Cu}(111)^{36}$ interfaces have shown that the first water layer has a structure with higher density than that of either ice or liquid water. A high-density form of interfacial water has also been identified for water on $\mathrm{NaCl}(001)$ in $\mathrm{MD}$ simulations ${ }^{37}$ as well as for water in nano-confinement ${ }^{13,21}$. In a recent study of the role of lattice mismatch in ice nucleation Cox et al. ${ }^{38}$ found that the angular flexibility in the second shell allowed closer packing at their model interface. These findings can now be interpreted in the context of the present results with the formation of a highly compressed, HDL-like phase at the interface. In fact, the HDL-like phase persists well below freezing without any signatures of ice formation in a way similar to the freezing point lowering seen in $\mathrm{NaCl}$ solutions ${ }^{39}$.

We have shown that the $\mathrm{BaF}_{2}(111)$ surface does not act as an ice formation template for ambient and supercooled water films, notwithstanding the close lattice match. The XAS spectrum of water on $\mathrm{BaF}_{2}(111)$, on the contrary, indicates limited tetrahedral H-bonding at $300-259 \mathrm{~K}$, where the similarity to spectra of high density ices suggests a highly compressed HDL-like water structure even in the supercooled regime. The $\mathrm{O}-\mathrm{O}$ pair-correlation function of the first water layer from MD simulations fully supports this high-density form of water at the interface.

\section{Methods}

Measurement techniques. Relative humidity-dependent water uptake curves were obtained using XPS to monitor the water coverage (see Supplementary Information for details). The $\mathrm{BaF}_{2}(111)$ surface was exposed to 1.5 Torr water vapor and the uptake was carefully controlled by reducing the sample temperature in an incremental fashion. 2.4 ML of water was the maximum coverage at $90 \% \mathrm{RH}$ and uptake curves are in good agreement with the ones measured using IR spectroscopy ${ }^{19}$.

The low temperature, UHV experiments on ice on $\mathrm{BaF}_{2}(111)$ were performed at the soft x-ray Spectroscopy Beamline (BL 13.2) at the Stanford Synchrotron Radiation Lightsource (SSRL). X-ray absorption spectra were acquired by the total electron yield (TEY) method. Ambient pressure XPS and XAS experiments were performed at the Molecular Environmental Science Beamline (BL 11.0.2) at the Advanced Light Source (ALS). The differentially pumped electron energy spectrometer (Specs Phobios 150) enables measurements at pressures up to $5 \mathrm{Torr}^{40}$. XAS data at 1.5 Torr $\mathrm{H}_{2} \mathrm{O}$ were acquired by the Auger electron yield (AEY) method using a kinetic energy detection window of $370-400 \mathrm{eV}$. Details of the sample preparation and data analysis are presented in figure captions and in the Supplementary Information.

MD simulations. MD simulations were performed to model the behavior of $\mathrm{H}_{2} \mathrm{O}$ on a fluorine-terminated $\mathrm{BaF}_{2}(111)$ surface $\left(256 \mathrm{BaF}_{2}, 500 \mathrm{H}_{2} \mathrm{O}\right)$. We used the GROMACS package ver. $4.5 .3^{41}$, the OPLS-AA force field ${ }^{42}$, and the TIP4P/2005 water model $^{43}$; results using alternative force-fields are discussed in the Supplementary Information. Each simulation was equilibrated for at least $5 \mathrm{~ns}$, generic thermodynamic properties were monitored to ensure equilibration. The production runs were performed in the NPT ensemble for $20 \mathrm{~ns}$ at a time step of 1 fs using velocity rescaling to set the temperature; the Berendsen barostat was applied for the pressure. All simulation results shown here were obtained at 1 bar and $290 \mathrm{~K}$ or $260 \mathrm{~K}$, respectively.

1. Debenedetti, P. G. Supercooled and glassy water. J. Phys. Condens. Mat. 15, R1669-R1726 (2003).

2. Finney, J. L., Hallbrucker, A., Kohl, I., Soper, A. K. \& Bowron, D. T. Structures of high and low density amorphous ice by neutron diffraction. Phys. Rev. Lett. 88, 225503-4 (2002).
3. Mishima, O., Calvert, L. D. \& Whalley, E. Melting ice I at $77 \mathrm{~K}$ and $10 \mathrm{kbar}$ : a new method of making amorphous solids. Nature 310, 393-395 (1984).

4. Finney, J. L. et al. Structure of a new dense amorphous ice. Phys. Rev. Lett. 89, 205503-4 (2002).

5. Loerting, T. et al. How many amorphous ices are there? Phys. Chem. Chem. Phys. 13, 8783-8794 (2011)

6. Tulk, C. A. et al. Structural studies of several distinct metastable forms of amorphous ice. Science 297, 1320-1323 (2002).

7. Kim, C. U., Barstow, B., Tate, M. W. \& Gruner, S. M. Evidence for liquid water during the high-density to low-density amorphous ice transition. Proc. Natl. Acad. Sci. USA 106, 4596-5 (2009).

8. Mishima, O. \& Stanley, H. E. The relationship between liquid, supercooled and glassy water. Nature 396, 329-335 (1998).

9. Capaccioli, S. \& Ngai, K. L. Resolving the controversy on the glass transition temperature of water? J. Chem. Phys. 135, 104504-12 (2011).

10. Seidl, M. et al. Volumetric study consistent with a glass-to-liquid transition in amorphous ices under pressure. Phys. Rev. B 83, 100201-4 (2011).

11. Petrenko, V. F. Physics of Ice (Oxford University Press, 1999).

12. Okhulkov, A. V., Demianets, Y. N. \& Gorbaty, Y. E. X-ray scattering in liquid water at pressures of up to $7.7 \mathrm{kbar}$ : Test of a fluctuation model. J. Chem. Phys. 100, 1578-1588 (1994).

13. Xu, S., Scherer, G. W., Mahadevan, T. S. \& Garofalini, S. H. Thermal Expansion of Confined Water. Langmuir 25, 5076-5083 (2009).

14. Du, Q., Freysz, E. \& Shen, Y. R. Vibrational spectra of water molecules at quartz/ water interfaces. Phys. Rev. Lett. 72, 238-241 (1994).

15. Asay, D. B. \& Kim, S. H. Evolution of the adsorbed water layer structure on silicon oxide at room temperature. J. Phys. Chem. B 109, 16760-16763 (2005).

16. Miranda, P. B., Xu, L., Shen, Y. R. \& Salmeron, M. Icelike water monolayer adsorbed on mica at room temperature. Phys. Rev. Lett. 81, 5876-5879 (1998).

17. Odelius, M., Bernasconi, M. \& Parrinello, M. Two dimensional ice adsorbed on mica surface. Phys. Rev. Lett. 78, 2855-2858 (1997).

18. Zink, J. C., Reif, J. \& Matthias, E. Water adsorption on (111) surfaces of $\mathrm{BaF}_{2}$ and $\mathrm{CaF}_{2}$. Phys. Rev. Lett. 68, 3595-3598 (1992).

19. Sadtchenko, V., Conrad, P. \& Ewing, G. E. $\mathrm{H}_{2} \mathrm{O}$ adsorption on $\mathrm{BaF}_{2}(111)$ at ambient temperatures. J. Chem. Phys. 116, 4293-4301 (2002).

20. Nutt, D. R. \& Stone, A. J. Adsorption of water on the $\mathrm{BaF}_{2}(111)$ surface. J. Chem. Phys. 117, 800 (2002).

21. Erko, M., Findenegg, G. H., Cade, N., Michette, A. G. \& Paris, O. Confinementinduced structural changes of water studied by Raman scattering. Phys. Rev. B 84, 104205-8 (2011).

22. Wassermann, B., Reif, J. \& Matthias, E. Different modes of water aggregation on (111) surfaces of fluorite structures. Phys. Rev. B 50, 2593-2597 (1994).

23. Conrad, P., Ewing, G. E., Karlinsey, R. L. \& Sadtchenko, V. Ice nucleation on $\mathrm{BaF}_{2}$ (111). J. Chem. Phys. 122, 064709-11 (2005).

24. Cardellach, M., Verdaguer, A., Santiso, J. \& Fraxedas, J. Two-dimensional wetting: The role of atomic steps on the nucleation of thin water films on $\mathrm{BaF}_{2}(111)$ at ambient conditions. J. Chem. Phys. 132, 234708-7 (2010).

25. Verdaguer, A., Cardellach, M. \& Fraxedas, J. Thin water films grown at ambient conditions on $\mathrm{BaF}_{2}(111)$ studied by scanning polarization force microscopy. J. Chem. Phys. 129, 174705-7 (2008).

26. Nilsson, A. et al. X-ray absorption spectroscopy and X-ray Raman scattering of water and ice; an experimental view. J. Electron. Spectrosc. Rel. Phenom. 177, 99-129 (2010).

27. Wernet, P. et al. The structure of the first coordination shell in liquid water. Science 304, 995-999 (2004).

28. Nilsson, A. \& Pettersson, L. G. M. Perspective on the structure of liquid water. Chem. Phys. 389, 1-34 (2011).

29. Tse, J. S. et al. X-ray Raman spectroscopic study of water in the condensed phases Phys. Rev. Lett. 100, 095502-4 (2008).

30. Pylkkänen, T. et al. Role of non-hydrogen-bonded molecules in the oxygen Kedge spectrum of ice. J. Phys. Chem. B 114, 3804-3808 (2010).

31. Waluyo, I. et al. The structure of water in the hydration shell of cations from $\mathrm{x}$-ray Raman and small angle $\mathrm{x}$-ray scattering measurements. J. Chem. Phys. 134, 064513-10 (2011).

32. Leberman, R. \& Soper, A. Effect of high-salt concentrations on water structure. Nature 378, 364-366 (1995).

33. Holzmann, J., Ludwig, R., Geiger, A. \& Paschek, D. Pressure and salt effects in simulated water: two sides of the same coin? Angew. Chem. Int. Ed. 46, 8907-8911 (2007).

34. Soper, A. K. \& Ricci, M. A. Structures of high-density and low-density water. Phys. Rev. Lett. 84, 2881-2884 (2000).

35. Schöder, S. et al. Radiation-induced premelting of ice at silica interfaces. Phys. Rev. Lett. 103, 095502-4 (2009).

36. Ito, M. Structures of water at electrified interfaces: Microscopic understanding of electrode potential in electric double layers on electrode surfaces. Surf. Sci. Rep. 63, 329-389 (2008).

37. Liu, L., Krack, M. \& Michaelides, A. Density oscillations in a nanoscale water film on salt: insight from ab initio molecular dynamics. J. Am. Chem. Soc. 130, 8572-8573 (2008).

38. Cox, S. J., Kathmann, S. M., Purton, J. A., Gillan, M. J. \& Michaelides, A. Nonhexagonal ice at hexagonal surfaces: the role of lattice mismatch. Phys. Chem. Chem. Phys. 14, 7944-7949 (2012). 
39. Bodnar, R. Revised equation and table for determining the freezing-point depression of $\mathrm{H}_{2} \mathrm{O}-\mathrm{NaCl}$ solutions. Geochim. Cosmochim. Ac. 57, 683-684 (1993).

40. Bluhm, H. Photoelectron spectroscopy of surfaces under humid conditions. J. Electron. Spectrosc. Rel. Phenom. 177, 71-84 (2010).

41. Hess, B., Kutzner, C., Van der Spoel, D. \& Lindahl, E. GROMACS 4: algorithms for highly efficient, load-balanced, and scalable molecular simulation. J. Chem. Theory Comput. 4, 435-447 (2008).

42. Jorgensen, W. L., Maxwell, D. S. \& Tirado-Rives, J. Development and testing of the opls all-atom force field on conformational energetics and properties of organic liquids. J. Am. Chem. Soc. 118, 11225-11236 (1996).

43. Abascal, J. L. F. \& Vega, C. A general purpose model for the condensed phases of water: TIP4P/2005. J. Chem. Phys. 123, 234505-12 (2005).

44. Mancinelli, R., Botti, A., Bruni, F., Ricci, M. A. \& Soper, A. K. Perturbation of water structure due to monovalent ions in solution. Phys. Chem. Chem. Phys. 9, 2959-2967 (2007)

\section{Acknowledgements}

This work was supported by the U.S. National Science Foundation, through grants CHE-043142 and CHE-0809324, by the Department of Energy, Office of Basic Energy Sciences, DE-AC02-76SF00515, and the Swedish Research Council. The measurements were carried out at the Stanford Synchrotron Radiation Lightsource (SSRL) and the Advanced Light Source (ALS), both national user facilities operated by Stanford University and U.C. Berkeley, respectively, on behalf of the U.S. Department of Energy, Office of Basic Energy Sciences. The ALS and the MES beamline 11.0.2 are supported by the Director, Office of Science, Office of Basic Energy Sciences, Division of Chemical Sciences,
Geosciences and Biosciences and Materials Sciences Division of the US Department of Energy at the Lawrence Berkeley National Laboratory under Contract No.

$\mathrm{DE}-\mathrm{AC} 02-05 \mathrm{CH} 11231$. The MD simulations were performed on resources provided by the Swedish National Infrastructure for Computing (SNIC) at the NSC center. J.T.N.

acknowledges the National Science Foundation for Postdoctoral fellowship support under ANT-1019347.

\section{Author contributions}

S.K. S.Y. and A.N planned, S.K., S.Y., J.T.N., H.B. and T.K. performed ambient pressure experiments, S.K. and H.O. performed vacuum based measurements. D.S. did the numerical simulations. The experimental data was analyzed by S.K. S.K., D.S., L.G.M.P. and A.N. wrote the paper. All authors participated in the discussions during data analysis and writing processes and reviewed the manuscript.

\section{Additional information}

Supplementary information accompanies this paper at http://www.nature.com/ scientificreports

Competing financial interests: The authors declare no competing financial interests.

License: This work is licensed under a Creative Commons

Attribution-NonCommercial-NoDerivs 3.0 Unported License. To view a copy of this license, visit http://creativecommons.org/licenses/by-nc-nd/3.0/

How to cite this article: Kaya, S. et al. Highly Compressed Two-Dimensional Form of Water at Ambient Conditions. Sci. Rep. 3, 1074; DOI:10.1038/srep01074 (2013). 